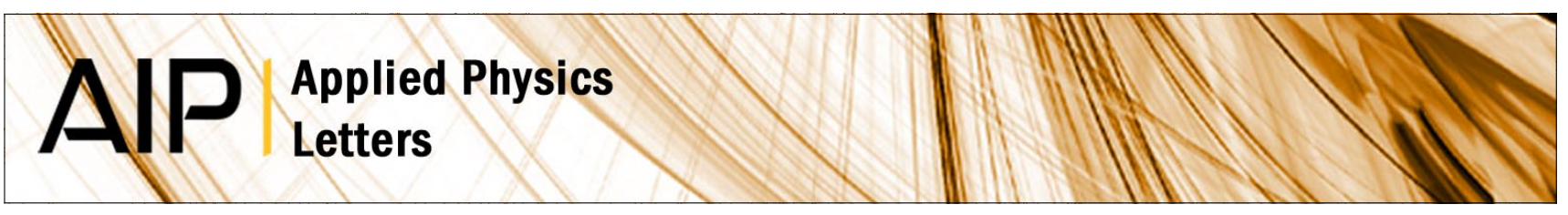

\title{
Spin excitation frequencies in magnetostatically coupled arrays of vortex state circular Permalloy dots
}
A. A. Awad, G. R. Aranda, D.
D. Dieleman,
an, K. Y. Guslienko,
G. N. Kakazei et al.

Citation: Appl. Phys. Lett. 97, 132501 (2010); doi: 10.1063/1.3495774

View online: http://dx.doi.org/10.1063/1.3495774

View Table of Contents: http://apl.aip.org/resource/1/APPLAB/v97/i13

Published by the American Institute of Physics.

\section{Related Articles}

Spin-polarized transport in zigzag-edge graphene nanoribbon junctions

J. Appl. Phys. 111, 014304 (2012)

Spin wave modes in ferromagnetic tubes

J. Appl. Phys. 111, 013905 (2012)

Spin-transfer mechanism for magnon-drag thermopower

Appl. Phys. Lett. 99, 262506 (2011)

Magnon magnetoresistance of NiFe nanowires: Size dependence and domain wall detection

Appl. Phys. Lett. 99, 262504 (2011)

Magnetic properties of $\mathrm{Fe}$ chains on $\mathrm{Cu} 2 \mathrm{~N} / \mathrm{Cu}(100)$ : A density functional theory study

J. Appl. Phys. 110, 123915 (2011)

\section{Additional information on Appl. Phys. Lett.}

Journal Homepage: http://apl.aip.org/

Journal Information: http://apl.aip.org/about/about_the_journal

Top downloads: http://apl.aip.org/features/most_downloaded

Information for Authors: http://apl.aip.org/authors

\section{ADVERTISEMENT}

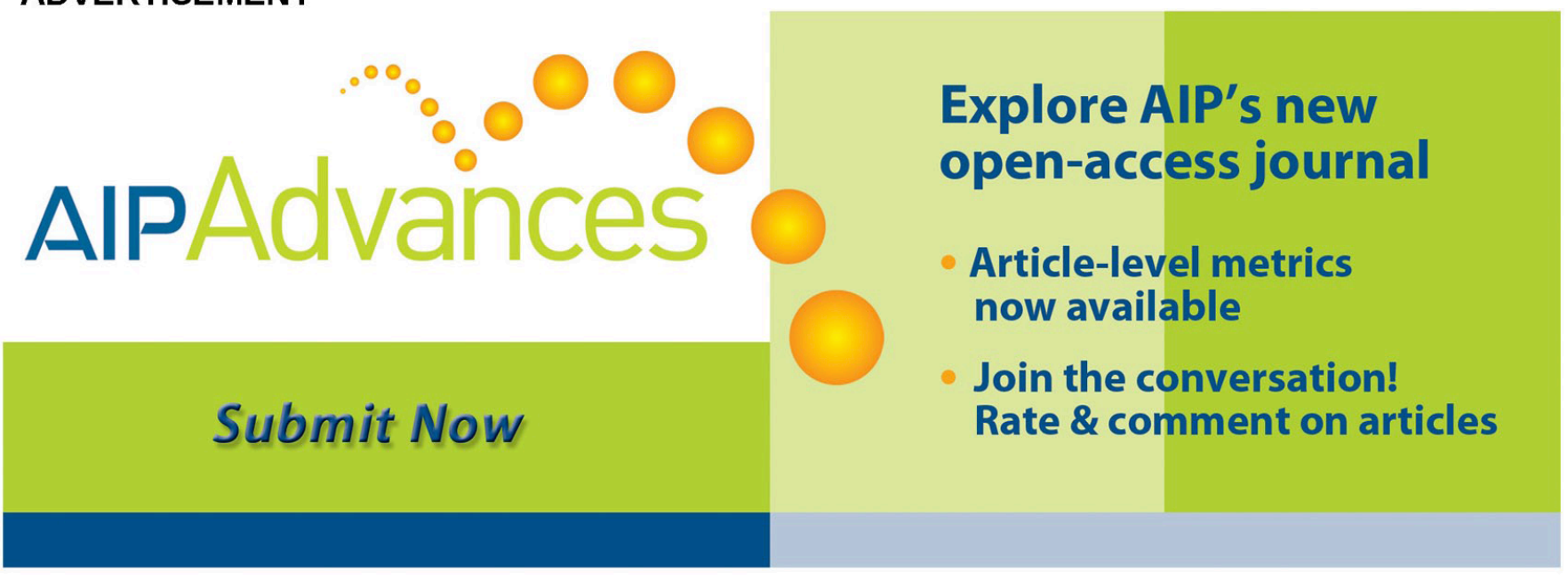




\title{
Spin excitation frequencies in magnetostatically coupled arrays of vortex state circular Permalloy dots
}

\author{
A. A. Awad, ${ }^{1}$ G. R. Aranda, ${ }^{2}$ D. Dieleman, ${ }^{1}$ K. Y. Guslienko, ${ }^{3, a)}$ G. N. Kakazei, ${ }^{4,5}$ \\ B. A. Ivanov, ${ }^{5}$ and F. G. Aliev ${ }^{1}$ \\ ${ }^{1}$ Dpto. Física de la Materia Condensada, CIII, Universidad Autónoma de Madrid, 28049 Madrid, Spain \\ ${ }^{2}$ Centro de Física de Materiales, CSIC, UPV/EHU, 20018 San Sebastián, Spain \\ ${ }^{3}$ Dpto. Fisica de Materiales, Universidad del Pais Vasco, 20018 Donostia-San Sebastian, Spain and \\ IKERBASQUE, The Basque Foundation for Science, 48011 Bilbao, Spain \\ ${ }^{4}$ Dpto. Fisica da Faculdade de Ciencias, IFIMUP and IN-Institute of Nanoscience and Nanotechnology, \\ Universidade do Porto, 4169-007 Porto, Portugal \\ ${ }^{5}$ Institute of Magnetism, National Academy of Sciences of Ukraine, 03142 Kiev, Ukraine
}

(Received 2 August 2010; accepted 9 September 2010; published online 28 September 2010)

\begin{abstract}
Broadband ferromagnetic resonance in square arrays of Permalloy circular dots with different interdot separations was measured in the vortex ground state. The detected spin excitations show a complicated dependence of their frequencies on the interdot coupling strength. A considerable influence of the interdot separation on the gyrotropic vortex frequency and splitting of the azimuthal spin wave frequencies was detected. The gyrotropic frequency and the first azimuthal doublet frequency splitting depend nonmonotonously on the interdot spacing, whereas the dependence of the second doublet frequency splitting on this parameter is monotonous. The observed effects are explained by the influence of both the dipolar and quadrupolar contributions to the dynamic magnetostatic interactions. (C) 2010 American Institute of Physics. [doi:10.1063/1.3495774]
\end{abstract}

Patterned nanomagnetic structures currently have great perspectives to be implemented as magnetic field and spin polarized current controlled devices in which spin excitations (magnons) are used to transfer and process information. It is well known that in arrays of uncoupled patterned elements (dots), the spin excitation spectra are quantized due to lateral confinement and standing spin waves can be excited and detected using different experimental techniques such as Brillouin light scattering (BLS), ferromagnetic resonance (FMR), time-resolved Kerr imaging, etc. The appearance of coupling between the dots in dense arrays decreasing interdot distances leads to an essential change in their spin excitation spectra.

In so-called magnonic crystals (MCs), ${ }^{1}$ based on either one-dimensional (1D) or two-dimensional (2D) periodically modulated magnetic structures, the artificially introduced periodicity and interelement coupling modify the spin excitation spectra in a controllable way. In particular, allowed and forbidden spin excitation frequency bands can appear. ${ }^{2}$ Most of the previous investigations of magnetization dynamics have been focused on interacting nano(micro)stripe arrays (1D MC), where the propagating spin waves were observed by means of BLS. ${ }^{3-5}$ Experimental investigations of the spin wave propagation in $2 \mathrm{D} \mathrm{MC}$ of square Permalloy dots using BLS (Ref. 6) indicated a strong dependence of the spin wave spectrum on the interdot distance. Influence of interdot coupling on the spectra in 2D MC of square arrays of circular dots using BLS was measured ${ }^{7}$ and the observed fourfold magnetic anisotropy was attributed to nonuniform magnetization of the dots. A general approach to this induced magnetic anisotropy was developed, ${ }^{8}$ and afterwards particular models $^{9-11}$ have been applied to explain the FMR experimental data obtained on 2D dot arrays.

This letter reports on influence of the magnetostatic in-

\footnotetext{
a) Author to whom correspondence should be addressed. Electronic mail: sckguslk@ehu.es.
}

terdot coupling on spin excitation spectra in the vortex ground state (in the absence of external magnetic field) of arrays of $1000 \mathrm{~nm}$ diameter Permalloy dots for a broad range of interdot separations $(d=200-1500 \mathrm{~nm})$. The precise probing of spin excitations of the arrays was conducted by broadband FMR. Static vortices do not generate stray fields except the stray fields localized in the small regions near the vortex cores. Therefore, in the absence of bias in-plane magnetic field, the sole source of the interdot coupling is the magnetostatic interaction of the dynamical magnetizations of the different dots.

Square arrays of Permalloy (Py, $\mathrm{Ni}_{80} \mathrm{Fe}_{20}$ ) circular dots were fabricated by combination of lithography and lift-off techniques on a standard $\mathrm{Si}(100)$ substrate with a $2.5 \mathrm{~nm}$ oxide layer on top, as explained in details elsewhere. ${ }^{12}$ The set consists of three samples with different lattice periods $a$ $=2 R+d$ of 1200,1500 , and $2500 \mathrm{~nm}$. The dot thickness $L$ is $50 \mathrm{~nm}$ and radius $R$ is $500 \mathrm{~nm}$. The excited in linear regime (in-plane driving rf field is below $0.2 \mathrm{Oe}$ ) spin modes have been studied in the frequency range from $100 \mathrm{MHz}$ up to 20 $\mathrm{GHz}$ at room temperature with high resolution $\sim 10 \mathrm{MHz}$, unavailable for the BLS and Kerr techniques, by using a broadband vector network analyzer-FMR spectrometer ${ }^{12}$ with a coplanar wave (CPW) guide in transmission configuration. The obtained data were analyzed on the basis of transmission model, see details in Ref. 13.

Multiple spin eigenmodes of a different nature can be excited in magnetic dots with a vortex ground state by an external perturbation. In the linear regime, the total dynamical response is described by a superposition of these modes. The excitation spectrum of a single circular dot in a vortex state consists of the low-frequency gyrotropic mode [typically well below $1 \mathrm{GHz}$ (Ref. 14)], and high frequency spin wave modes of different symmetry. These are labeled by an azimuthal number, $m$, and the number of nodes of dynamical magnetization, $n$, along the dot radial direction. ${ }^{15-17}$ The gyrotropic modes with $|m|=1$ are associated with the displace- 

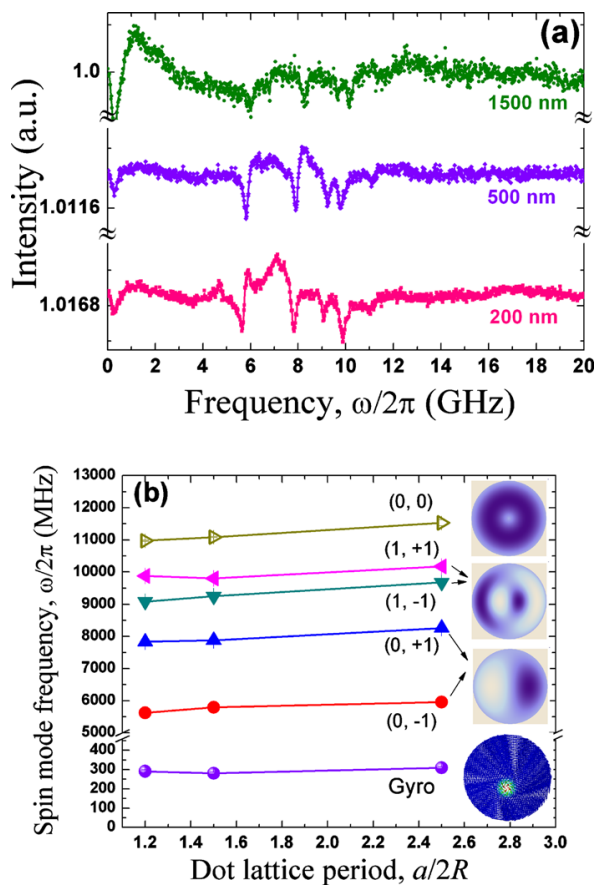

FIG. 1. (Color online) (a) Zero in-plane bias field response of the three arrays of Py dots with different interdot separations, $d=200,500$, and 1500 $\mathrm{nm}$. (b) The detected spin excitation frequencies labeled by the mode indices $(n, m)$ vs the interdot separation and the $(n, m)$ spin wave modes and gyrotropic mode images. The dynamic magnetization of the $(n, \pm 1)$ modes rotates clockwise for $m=-1$ or counter-clockwise for $m=+1$.

ment of the vortex core from the dot center. Due to topology of the vortex, the frequencies of azimuthal modes $(m \neq 0)$ are splitted in doublets having indices $m= \pm|m|$, with the largest value of splitting for $|m|=1 .{ }^{17-19}$ For isolated micron and submicron sized soft magnetic dots, the spin wave eigenmode frequencies are determined by the dot aspect ratio $\beta$ $=L / R$, and are proportional to $\sqrt{\beta}$, whereas the gyrotropic frequency and the $m= \pm 1$ doublet splitting are linear on $\beta$ for thin dots $(\beta \ll 1){ }^{19,20}$

Figure 1(a) shows the variation in the modulus of the transmitted rf signal at zero magnetic field (the centered vortex ground state), indicating the above-mentioned spin eigenmodes that are excited by uniform rf field. A similar set of the spin eigenmodes was observed in all the samples under study; a gyrotropic mode, two doublets of the high frequency azimuthal spin wave modes with $n=0,1$ and $m= \pm 1$, and one low-intensity radial mode indexed as $n=0, m=0$. Estimation on the basis of equations of Ref. 15 yields for this frequency the value of $12.7 \mathrm{GHz}$. The influence of the interdot separation on the observed resonance peak positions is well noticeable for all the modes [Fig. 1(b)]. It is most significant for the gyrotropic mode frequency $\omega_{g}$, and for the values of the frequency splitting for $m= \pm 1$ doublets with $n=0$ and $n=1, \Delta \omega_{0}$, and $\Delta \omega_{1}$, respectively (Fig. 2). Both the gyrotropic frequency and the splitting $\Delta \omega_{0}$ reveal wellpronounced nonmonotonous dependence on the dot array period $a$, whereas the dependence $\Delta \omega_{1}(a)$ is monotonous.

To explain this complicated behavior, we consider the interdot coupling of the ground vortex state dots within an array. The only source of the coupling in our case is the magnetostatic interaction of the nonuniform dynamical magnetizations $\mathbf{m}(\mathbf{r}, t)$ of the different dots. For an array of well separated dots, $R \ll a$, a theory of the collective spin modes has been developed by expansion of the magnetostatic en-

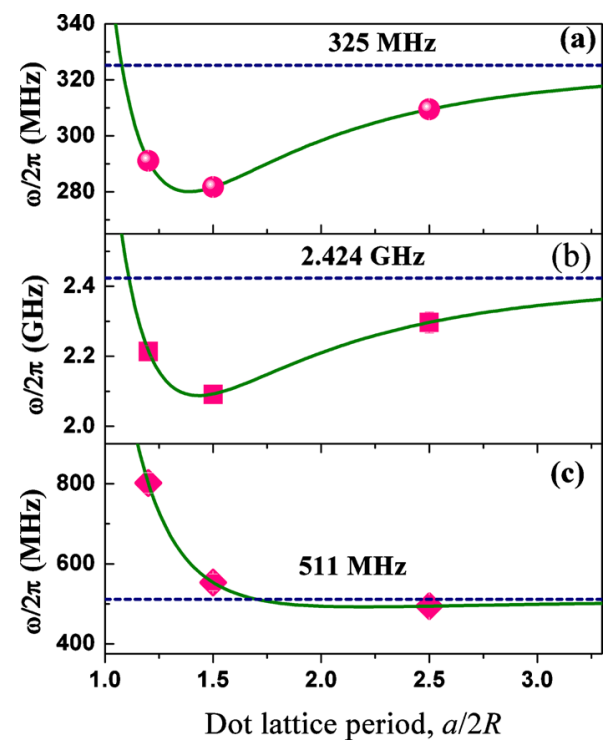

FIG. 2. (Color online) The dependence of the gyrotropic frequency $\omega_{g}$ (a) and the frequency splitting of the first $\Delta \omega_{0}$ (b) and the second $\Delta \omega_{1}$ (c) doublets of azimuthal spin waves on the dot array period $a$. Symbols represent the FMR experimental data, solid green lines give the description of the experimental data by Eq. (1). Horizontal dotted lines present the limit frequency values $\omega_{\alpha}(0)$ found for isolated dot, $(R / a) \rightarrow 0$.

ergy over the small parameter $R / a .^{21}$ Within this approach, the interdot interaction can be presented as a sum of different multipole contributions similar to a multipole expansion in classical electrodynamics. The first (dipolar) term is determined by oscillations of the total dot magnetic moments $\boldsymbol{\mu}(t)=\int d \mathbf{r m}(\mathbf{r}, t)$. This dipolar term leads to contributions to the eigenfrequencies that are proportional to $1 / a^{3}$. The nondipolar terms are determined mainly by the dot quadrupolar moments $q_{i j}=\int d \mathbf{r} m_{i} x_{j}, i, j=x, y, z .{ }^{8}$ The quadrupolar terms lead to $1 / a^{5}$ dependence of the interdot interaction energy. Only the spin modes with $m=0, \pm 1$ having nonzero $\boldsymbol{\mu}$ are affected by the interdot dipolar interactions. For the rest of the modes (with higher $m$ ) the sole source of the coupling is a nondipolar coupling. On the other hand, the quadrupolar interaction can be essential even for the dipolarly-active modes (with $m=0, \pm 1$ ). ${ }^{21}$

The particular excitation scheme of the FMR experiment, using an almost uniform in-plane rf field, corresponds to excitations of the modes with $m= \pm 1$ only, i.e., the gyrotropic mode and azimuthal spin waves. However, a small out-of-plane component of the rf field due to the finite CPW lateral size results in excitation of a low-intensity radial mode $(m=0, n=0) .{ }^{20}$ For our samples the parameter $2 R / a$ is not small (it varies from 0.4 to 0.83 ). Nevertheless, our experimental data can be explained taking into account both the dipolar and quadrupolar interactions of the modes. Within this simple scheme, the spin eigenfrequencies can be described by the equation

$$
\omega_{\alpha}=\omega_{\alpha}(0)-\Omega_{\alpha, d}\left(\frac{2 R}{a}\right)^{3}+\Omega_{\alpha, q}\left(\frac{2 R}{a}\right)^{5}
$$

where $\omega_{\alpha}$ with $\alpha=g, 0,1$ give the values of $\omega_{g}, \Delta \omega_{0}$, and $\Delta \omega_{1}$, respectively. The quantities $\omega_{\alpha}(0)$ describe the frequency values for noninteracting dots, and the parameters $\Omega_{\alpha, d}$ and $\Omega_{\alpha, q}$ are the weights of the dipolar and quadrupolar contributions to the corresponding frequencies. We discuss 
the experimental data on the ground of Eq. (1), with proper choice of the parameters $\Omega_{\alpha, d}$ and $\Omega_{\alpha, q}$.

First, we note that for $n=1, m= \pm 1$ azimuthal modes the amplitude of the dipolar moment $\boldsymbol{\mu}_{n, m}$ is much less than for $n=0, m= \pm 1$ modes due to radial node of the eigenfunctions, the corresponding ratio is $\mu_{1, \pm 1} / \mu_{0, \pm 1} \approx 0.13$. $^{21}$ Thus, the dipolar interaction is small for $n=1$ modes being of order of $2 \%$ of the value expected for $n=0$ modes. The quadrupolar interaction should be dominating for frequencies of the azimuthal modes with $n=1, m= \pm 1$, and their frequency splitting. The observed value of $m= \pm 1, n=1$ doublet splitting is well described by the dependence $\Delta \omega_{1}=\Delta \omega_{1}(0)$ $+\Omega_{1, q}(2 R / a)^{5}$, with positive constant $\Omega_{1, q}$ [Fig. 2(c)]. Thus, the quadrupolar interaction provides the upward shift in the frequencies for $n=1, m= \pm 1$ modes proportional to $(2 R / a)^{5}$.

For both the gyrotropic mode, and the main spin wave frequency doublet $(n=0,|m|=1)$ splitting, only the dipolar interaction was previously calculated giving the negative contribution with $\Omega_{\mathrm{g}, d}=\pi \beta \gamma M_{s} \sigma(0) / 72,{ }^{21}$ where $\sigma(0)$ $\approx 9.03$ is the dipolar sum, $\gamma$ is the gyromagnetic ratio, and $M_{s}$ is the saturation magnetization. Combining this dipolar contribution with the positive quadrupolar one of the same form as for $n=1$, a good description of the experimental data for $\omega_{g}$ and $\Delta \omega_{0}$ dependencies on $2 R / a$ is obtained, see Figs. 2(a) and 2(b). Here the nonmonotonous dependence of the frequencies on $a$ naturally appears as a competition of two contributions, dipolar and quadrupolar, with different signs. Most intriguing is the experimental observation that for the coupled dot arrays the proportionality $\Delta \omega_{0}(a) \propto \omega_{g}(a)$ holds.

Thus, Eq. (1) with proper choice of the coefficients $\Omega_{\alpha, d}$ and $\Omega_{\alpha, q}$ provides a good qualitative description for all the experimental data. As to a quantitative description of the data, the limiting value of $\omega_{g} / 2 \pi=325 \mathrm{MHz}$ [Fig. 2(a)] is somewhat lower than $480 \mathrm{MHz}$ expected from analytical theory ${ }^{14}$ and from our micromagnetic simulations (391 MHz) on an isolated Py dot with $R=500 \mathrm{~nm}$ and $L=50 \mathrm{~nm}$. ${ }^{22}$ The simulated first azimuthal doublet $(n=0, m= \pm 1)$ frequencies $6.35 \mathrm{GHz}$ and $8.40 \mathrm{GHz}$ are very close to the experimentally detected frequencies $5.96 \mathrm{GHz}$ and $8.26 \mathrm{GHz}$ at $d=1500 \mathrm{~nm}$ confirming their interpretation. The value of the dipolar interaction contribution to the gyrotropic frequency $\omega_{g}$ is much higher than in the theory. ${ }^{21}$ The ratio between the measured values of the $n=0, m= \pm 1$ doublet frequency splitting $\Delta \omega_{0}(2.09-2.30 \mathrm{GHz})$ and gyrotropic frequency (282-310 MHz), namely, $\Delta \omega_{0} / \omega_{g} \approx 7.4$, is twice higher than the ratio $\Delta \omega_{0} / \omega_{g} \approx 4$ calculated in Ref. 19. These discrepancies may be attributed to the fact that the corresponding calculations were performed in the main approximation on the small parameters, $\beta$ and $R / a$, whereas these ratios are not small in our case. For example, the value of $\beta=0.1$ is higher than in previous experiments. ${ }^{14,17}$ Note that the essential downward deviation from the linear dependence $\omega_{g}$ on $\beta$ was reported $^{23}$ for $\beta>0.1$ due to a nonuniformity of $\mathbf{m}$ along the dot thickness. One more possible source of such discrepancies is lowering of the symmetry of the vortex ground state magnetization distribution for the dense dot arrays, as well as different directions of the vortex chiralities and core polarizations.

To conclude, the effect of the interdot coupling on spin excitation frequencies in the vortex ground state of $2 \mathrm{D}$ dot arrays is quite different from that for saturated dots. Our measurements demonstrate the essential role of the dynami- cal magnetostatic interaction on the vortex dot spectra. Especially, the fine structure (the values of $\omega_{g}, \Delta \omega_{0}$, and $\Delta \omega_{1}$ ) of the spectra is very sensitive to the interdot coupling. Accounting both the dipolar and quadrupolar contributions yields a good qualitative description of the complicated spin excitation spectra in coupled dot arrays. The obtained results can serve as a basis for understanding of the propagation of spin excitations in 2D MC consisting of the vortex state magnetic dot arrays.

The work in Madrid was supported by Spanish MICINN (Grant Nos. MAT2009-10139, MAT2006-28183-E, and CSD2007-00010) and CAM (Grant No. S-505/MAT0194). K.Y.G. and G.R.A. acknowledge support by IKERBASQUE (the Basque Science Foundation), JAE-doc grant and the SAIOTEK Grant No. S-PC09UN03. G.N.K. acknowledges support from Portuguese FCT through the "Ciencia 2007" program. The work in Kiev was supported by the STCU Grant No. 5210 and the Grant No. 220-10 from NAS of Ukraine.

${ }^{1}$ V. Kruglyak, S. O. Demokritov, and D. Grundler, J. Phys. D: Appl. Phys. 43, 264001 (2010)

${ }^{2}$ K. Y. Guslienko, Phys. Solid State 37, 870 (1995); V. V. Kruglyak and R. J. Hicken, J. Magn. Magn. Mater. 306, 191 (2006).

${ }^{3}$ M. Kostylev, P. Schrader, R. L. Stamps, G. Gubbiotti, G. Carlotti, A. O. Adeyeye, S. Goolaup, and N. Singh, Appl. Phys. Lett. 92, 132504 (2008).

${ }^{4}$ A. Chumak, A. Serga, B. Hillebrands, and M. Kostylev, Appl. Phys. Lett. 93, 022508 (2008).

${ }^{5}$ G. Gubbiotti, S. Tacchi, G. Carlotti, N. Singh, S. Goolaup, A. O. Adeyeye, and M. Kostylev, Appl. Phys. Lett. 90, 092503 (2007).

${ }^{6}$ S. Tacchi, M. Madami, G. Gubbiotti, G. Carlotti, H. Tanigawa, T. Ono, and M. P. Kostylev, Phys. Rev. B 82, 024401 (2010).

${ }^{7}$ C. Mathieu, C. Hartmann, M. Bauer, O. Buettner, S. Riedling, B. Roos, S O. Demokritov, B. Hillebrands, B. Bartenlian, C. Chappert, D. Decanini, F. Rousseaux, E. Cambril, A. Müller, B. Hoffmann, and U. Hartmann, Appl. Phys. Lett. 70, 2912 (1997).

${ }^{8}$ K. Y. Guslienko, Phys. Lett. A 278, 293 (2001).

${ }^{9}$ K. L. Metlov, Phys. Rev. Lett. 97, 127205 (2006).

${ }^{10}$ G. N. Kakazei, Y. G. Pogorelov, M. D. Costa, T. Mewes, P. E. Wigen, P. C. Hammel, V. O. Golub, T. Okuno, and V. Novosad, Phys. Rev. B 74 060406 (2006).

${ }^{11}$ K. Rivkin, W. Xu, L. E. DeLong, V. V. Metlushko, B. Ilic, and J. B. Ketterson, J. Magn. Magn. Mater. 309, 317 (2007).

${ }^{12}$ F. G. Aliev, J. F. Sierra, A. A. Awad, G. N. Kakazei, D. S. Han, S. K. Kim, V. Metlushko, B. Ilic, and K. Y. Guslienko, Phys. Rev. B 79, 174433 (2009).

${ }^{13}$ J. F. Sierra, A. A. Awad, G. N. Kakazei, F. J. Palomares, and F. G. Aliev, IEEE Trans. Magn. 44, 3063 (2008).

${ }^{14}$ K. Y. Guslienko, B. A. Ivanov, V. Novosad, Y. Otani, H. Shima, and K Fukamichi, J. Appl. Phys. 91, 8037 (2002); V. Novosad, F. Y. Fradin, P. E. Roy, K. S. Buchanan, K. Y. Guslienko, and S. D. Bader, Phys. Rev. B 72, 024455 (2005).

${ }^{15}$ K. Y. Guslienko, W. Scholz, R. W. Chantrell, and V. Novosad, Phys. Rev. B 71, 144407 (2005).

${ }^{16}$ M. Buess, T. P. J. Knowles, R. Höllinger, T. Haug, U. Krey, D. Weiss, D. Pescia, M. R. Scheinfein, and C. H. Back, Phys. Rev. B 71, 104415 (2005).

${ }^{17}$ J. P. Park and P. A. Crowell, Phys. Rev. Lett. 95, 167201 (2005).

${ }^{18}$ B. A. Ivanov, H. J. Schnitzer, F. G. Mertens, and G. M. Wysin, Phys. Rev. B 58, 8464 (1998).

${ }^{19}$ K. Y. Guslienko, A. N. Slavin, V. T. Tiberkevich, and S.-K. Kim, Phys. Rev. Lett. 101, 247203 (2008).

${ }^{20}$ A. A. Awad, K. Y. Guslienko, J. F. Sierra, G. N. Kakazei, V. Metlushko, and F. G. Aliev, Appl. Phys. Lett. 96, 012503 (2010).

${ }^{21}$ A. Y. Galkin, B. A. Ivanov, and C. E. Zaspel, Phys. Rev. B 74, 144419 (2006).

${ }^{22} \mathrm{http} / / /$ math.nist.gov/oommf. The parameters were $M_{s}=800 \mathrm{emu} / \mathrm{cc}$, the exchange stiffness $A=1.3 \times 10^{-11} \mathrm{~J} / \mathrm{m}, \gamma / 2 \pi=2.95 \mathrm{MHz} / \mathrm{Oe}$, the damping $\alpha=0.01$, and in-plane cell size $5 \mathrm{~nm}$.

${ }^{23}$ F. Boust and N. Vukadinovic, Phys. Rev. B 70, 172408 (2004). 